\title{
Stability Assessment Approach Based on Trajectory Section Eigenvalue
}

\author{
Jiacheng Ruan ${ }^{1}$, Hengxu Zhang ${ }^{1 *}$, Yongji Cao ${ }^{1}$ \\ ${ }^{1}$ Key Laboratory of Power System Intelligent Dispatch and Control of the Ministry of Education, Shandong University, Jinan 250061, \\ China
}

\begin{abstract}
Eigenvalue Method can analyze the operating features of the system. The trajectory section eigenvalue method combines the model and trajectory, and extends the equilibrium point eigenvalue to the unbalanced point of the cross section at any time on the disturbed trajectory, which well solves the nonlinearity and the time-varying issues of the system. Based on the theoretical basis of clarifying the trajectory section eigenvalue (TSE) method, the paper establishes a stability evaluation framework based on the timevarying second-order resonant circuit, which extrates the damping feature and frequency feature information in the eigenvalue sequence of the trajectory section, then compares it with the results of signal processing. The influence mechanism of time-varying factors on system stability is clarified, and the advantages of the trajectory section eigenvalue method in stability evaluation are demonstrated.
\end{abstract}

\section{INTRODUCTION}

The state matrix of the linear time-invariant system is determined by the system itself and is not affected by external disturbances. The state matrix can reflect the motion features and global stability of the linear timeinvariant system and easy to solved in the time domain. The dynamic response of the system after being disturbed at the equilibrium point is completely determined by the state matrix, and the equilibrium point eigenvalue method is based on this. However, most of the actual engineering systems are time-varying and nonlinear, and the response of the system after being disturbed is difficult to solve in the time domain.

The common solution is to obtain an overall stable and structurally consistent linear model of the system at the system equilibrium point through the first-order Taylor expansion, construct the linear differential equation of the system to obtain the state matrix, and then analyze the dynamic features (features) of the system by obtaining the eigenvalue, however, it cannot account for time-varying and non-linear factors outside the equilibrium point, reflect the global dynamic features accurately [1].The shortcoming that equilibrium point eigenvalue can only be limited to the equilibrium point is revealed. To solve this problem, many scholars have done tentative work, trying to extend the equilibrium point eigenvalue method to the nonlinear region of the system. The vector field normal form method [2]-[5] and the modal series method [6], [7] are common methods. Both of them approximate the nonlinear factors in the system model by taking into account the higher-order Taylor expansion term of the nonlinear equation at the equilibrium point. However, these methods are limited to calculation feasibility, only take the second-order terms into account, and it is difficult to fully take into account the influence of strong nonlinear factors. In essence, these methods are still based on the system equation at the equilibrium point, and do not go beyond the limitations of the origin feature root method. It is still difficult to deal with strong nonlinear and time-varying factors while increasing calculation. Literature [8] even pointed out that if the system has time-delay links or discontinuous factors, the Taylor series will not converge, and the system model cannot be linearized at the equilibrium point. Under this theoretical predicament, related scholars pioneered the trajectory section eigenvalue [9], extending the eigenvalue method from the equilibrium point to the non-equilibrium point. Since then, the trajectory section eigenvalue has been widely concerned and applied [10]-[16]. Most research is devoted to mining information in time sections to complete clustering, disturbance determination, or local stability evaluation.

Low-frequency oscillation is one of the key issues of power system stability. Commonly used signal processing methods are used for identification and analysis, including wavelet method, Prony method and Hilbert-Huang Transform (HHT) method, etc. They each have good performance in accuracy, rapidity, stability and completeness, but they all have unavoidable limitations. Author will clarify the theoretical basis of the trajectory section eigenvalue, then use the eigenvalue information of the trajectory section and signal processing methods to reveal the dynamic response features of the time-varying system, based on the secondorder resonant circuit. In the end, the paper complete

\footnotetext{
*Corresponding author's e-mail: zhanghx@sdu.edu.cn
} 
the evaluation of the system stability, compare the performance of the trajectory section eigenvalue (TSE) method and the signal processing method on stability online evaluation work.

\section{Theory Of Trajectory Section EigenVALUE}

Literature [17] pointed out that Euler integration means that the system dynamics in each analysis step on the disturbed trajectory can be approximated by a linear function. Therefore, the mathematical model of the system in the analysis step can be approximated as a linear steady system with external excitation. The system state matrix is determined by the topological structure and algebraic value of the initial time section, the timevarying nonlinear system can be equivalent to a piecewise linear time-invariant system, as long as the integration step length is reasonable and without strong time-varying nonlinear factors in each integration step. The linear model needs to be revised at every analysis step, including algebraic value, external excitation, dimension, etc.

\subsection{Piecewise Linearization}

Describe nonlinear system models with differentialalgebraic equations

$$
\left\{\begin{array}{l}
\dot{X}=f(X, Y) \\
0=g(X, Y)
\end{array}\right.
$$

$\boldsymbol{X}$ represents the system state variable, and $\boldsymbol{Y}$ represents the system algebraic variable. The updated value of each time interval state variable and algebraic variable is obtained by numerical integration.

$$
\begin{aligned}
& \frac{\mathrm{d}\left(\boldsymbol{X}\left(t_{k}\right)+\Delta \boldsymbol{X}_{k}(\Delta t)\right)}{\mathrm{d}\left(t_{k}+\Delta t\right)}=\boldsymbol{f}\left(\boldsymbol{X}\left(t_{k}\right), \boldsymbol{Y}\left(t_{k}\right)\right)+ \\
& \left.\frac{\partial \boldsymbol{f}}{\partial \boldsymbol{X}}\right|_{\boldsymbol{X}\left(t_{k}\right)} \Delta \boldsymbol{X}_{k}(\Delta t)+\left.\frac{\partial \boldsymbol{f}}{\partial \boldsymbol{Y}}\right|_{\boldsymbol{Y}\left(t_{k}\right)} \Delta \boldsymbol{Y}_{k}(\Delta t)
\end{aligned}
$$$$
\boldsymbol{X}\left(t_{k}\right) \text { and } \boldsymbol{Y}\left(t_{k}\right) \text { represents the state variable }
$$
increment and the algebraic variable increment respectively. $\boldsymbol{f}\left(\boldsymbol{X}\left(t_{k}\right), \boldsymbol{Y}\left(t_{k}\right)\right)$ is the initial value of the state variable and algebraic variable at the $k$ time section, can be expressed as $\boldsymbol{B}_{k}$ and regarded as an external stimulus. Equation (2) can be simplified to

$$
\Delta \dot{\boldsymbol{X}}_{k}(\Delta t)=\boldsymbol{A}_{k} \Delta \boldsymbol{X}_{k}(\Delta t)+\boldsymbol{B}_{k}
$$

Equation (3) transforms the nonlinear differential equations into linear differential equations with timevarying disturbance terms, completes the continuation of the equilibrium point eigenvalue method on the nonequilibrium point.

\subsection{State Incremental Reconstruction Based on Trajectory Section Eigenvalues}

In order to prove the correctness of the eigenvalue method of the trajectory section, the following changes are made to formula

$$
\boldsymbol{Z}_{k}(\Delta t)=\boldsymbol{U}_{k}^{-1} \Delta \boldsymbol{X}_{k}(\Delta t)
$$

$\boldsymbol{U}_{k}$ and $\boldsymbol{U}_{k}^{-1}$ are the modal matrix and the modal inverse matrix respectively, formula can be obtained after transformation.

$$
\dot{\boldsymbol{Z}}_{k}(\Delta t)=\boldsymbol{U}_{k}^{-1} \boldsymbol{A}_{k} \boldsymbol{U}_{k} \boldsymbol{Z}_{k}(\Delta t)+\boldsymbol{U}_{k}^{-1} \boldsymbol{B}_{k}
$$

The time domain solution is as follows

$$
\boldsymbol{Z}_{k}(\Delta t)=\boldsymbol{C}_{k} \boldsymbol{T}_{k}(\Delta t)-\boldsymbol{\Lambda}_{k}^{-1} \boldsymbol{U}_{k}^{-1} \boldsymbol{B}_{k}
$$

$\boldsymbol{T}_{k}(\Delta t)$ is the exponential component representing the feature mode, $\boldsymbol{C}_{k}$ is the parameter matrix, can be obtained by $Z_{k}(0)=0$.

$$
\begin{gathered}
\boldsymbol{T}_{k}(\Delta t)=\left[e_{k}^{\lambda_{1} \Delta t}, e_{k}^{\lambda_{2} \Delta t}, e_{k}^{\lambda_{3} \Delta t}, e_{k}^{\lambda_{4} \Delta t} \cdots \cdots e_{k}^{\lambda_{n} \Delta t}\right] \\
\boldsymbol{C}_{k} \boldsymbol{T}_{k}(0)=\boldsymbol{\Lambda}_{k}^{-1} \boldsymbol{U}_{k}^{-1} \boldsymbol{B}_{k}
\end{gathered}
$$

Weighted reorganization of $\boldsymbol{Z}_{k}(\Delta t)$ from formula (6)

$$
\Delta \boldsymbol{X}_{k}(\Delta t)=\boldsymbol{U}_{k} \boldsymbol{C}_{k} \boldsymbol{T}_{k}(\Delta t)-\boldsymbol{U}_{k} \boldsymbol{\Lambda}_{k}^{-1} \boldsymbol{U}_{k}^{-1} \boldsymbol{B}_{k}
$$

Formula (11) is equal to formula (11)

$$
\Delta \boldsymbol{X}_{k}(\Delta t)=\boldsymbol{U}_{k} \boldsymbol{C}_{k} \boldsymbol{T}_{k}(\Delta t)-\boldsymbol{U}_{k} \boldsymbol{C}_{k} \boldsymbol{T}_{k}(0)
$$

make $\boldsymbol{t}^{=\Delta \boldsymbol{t}+\boldsymbol{t}_{\boldsymbol{k}}}$, at any time between time $\boldsymbol{t}_{\boldsymbol{k}}$ and $\boldsymbol{t}_{\boldsymbol{k}+1}$, the state increment expression is:

$$
\Delta \boldsymbol{X}_{k}\left(t-t_{k}\right)=\boldsymbol{U}_{k} \boldsymbol{C}_{k} \boldsymbol{T}_{k}\left(t-t_{k}\right)-\boldsymbol{U}_{k} \boldsymbol{C}_{k} \boldsymbol{T}_{k}(0)
$$

The incremental reconstruction of the state in the analysis step is completed.

\subsection{Error Analysis of State Incremental Reconstruction}

First, Tyler expands $\boldsymbol{T}_{k}(\Delta t)$ at $\Delta t=0$

$$
\begin{aligned}
& \boldsymbol{T}_{k}(\Delta t)=\boldsymbol{T}_{k}(0)+\boldsymbol{\Lambda}_{k} \boldsymbol{T}_{k}(0) \Delta t \\
& +\boldsymbol{\Lambda}_{k}^{2} \boldsymbol{T}_{k}(0) \xi^{2} / 2 ; \xi \in[0, \Delta t)
\end{aligned}
$$

Incorporate formula (12) into formula (9)

$$
\begin{array}{r}
\Delta \boldsymbol{X}_{k}(\Delta t)=\boldsymbol{U}_{k} \boldsymbol{C}_{k} \boldsymbol{T}_{k}(0)+\boldsymbol{U}_{k} \boldsymbol{C}_{k} \boldsymbol{\Lambda}_{k} \boldsymbol{T}_{k}(0) \Delta t \\
+\boldsymbol{U}_{k} \boldsymbol{C}_{k} \boldsymbol{\Lambda}_{k}^{2} \boldsymbol{T}_{k}(0) \xi^{2} / 2-\boldsymbol{U}_{k} \boldsymbol{\Lambda}_{k}^{-1} \boldsymbol{U}_{k}^{-1} \boldsymbol{B}_{k}
\end{array}
$$

Formula (14) is simplified from (13)

$$
\Delta \boldsymbol{X}_{k}(\Delta t)=\boldsymbol{U}_{k} \boldsymbol{C}_{k} \boldsymbol{\Lambda}_{k} \boldsymbol{T}_{k}(0) \Delta t+\boldsymbol{U}_{k} \boldsymbol{C}_{k} \boldsymbol{\Lambda}_{k}^{2} \boldsymbol{T}_{k}(0) \xi^{2} / 2
$$

Given that

$$
\boldsymbol{U}_{k} \boldsymbol{C}_{k} \boldsymbol{\Lambda}_{k} \boldsymbol{T}_{k}(0)=\boldsymbol{B}_{k}=\boldsymbol{f}\left(\boldsymbol{X}\left(t_{k}\right), \boldsymbol{Y}\left(t_{k}\right)\right)
$$

further simplify

$$
\Delta \boldsymbol{X}_{k}(\Delta t)=\boldsymbol{f}\left(\boldsymbol{X}\left(t_{k}\right), \boldsymbol{Y}\left(t_{k}\right)\right) \Delta t+\boldsymbol{U}_{k} \boldsymbol{C}_{k} \boldsymbol{\Lambda}_{k}^{2} \boldsymbol{T}_{k}(0) \xi^{2} / 2
$$

The local truncation error of the reconstruction from $t_{k}$ to $t_{k+1}$ is

$$
\boldsymbol{R}(\Delta t)=\boldsymbol{U}_{k} \boldsymbol{C}_{k} \boldsymbol{\Lambda}_{k}^{2} \boldsymbol{T}_{k}(0) \xi^{2} / 2
$$
Euler 


$$
\Delta \boldsymbol{X}(\Delta t)=\boldsymbol{f}\left(\boldsymbol{X}\left(t_{k}\right), \boldsymbol{Y}\left(t_{k}\right)\right) \Delta t+\ddot{\boldsymbol{X}}\left(t_{k}\right) \xi^{2} / 2 ; \xi \in[0, \Delta t)
$$

The local truncation error of the first-order Euler method is

$$
\boldsymbol{P}(\Delta t)=\ddot{\boldsymbol{X}}\left(t_{k}\right) \xi^{2} / 2
$$

$\boldsymbol{R}(\Delta t)$ and $\boldsymbol{P}(\Delta t)$ are infinitesimals of the same order, when the local truncation error of the first-order Euler method is bounded, the local truncation error of the reconstruction increment of the trajectory section eigenvalue is also bounded. Therefore, by setting $\Delta t$, the local truncation error of the reconstruction can be approached to zero. The trajectory section eigenvalue method updates the state matrix at each analysis step, without accumulated error.

\section{TRAJECTORY SECTION EIGENVALUE OF SECOND-ORDER RESONANT CIRCUIT}

There are two independent energy storage elements in the classic second-order resonant circuit, the capacitor $\mathrm{C}$ and the inductance L, so there are two state variables. The selection of state variables is arbitrary in principle [18], choose $u_{c}$ and $i$ as the two state variables of this system.

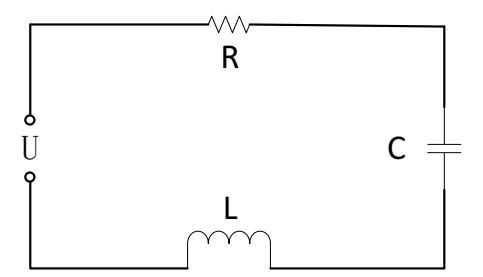

Figure 1 Second order resonant circuit

The mathematical model of the second-order resonant circuit is as follows

$$
\left\{\begin{array}{l}
\frac{d U_{c}}{d t}=\frac{1}{C} I \\
\frac{d I}{d t}=-\frac{U_{c}}{L}-\frac{R}{L} I+\frac{U_{r}}{L}
\end{array}\right.
$$

State space expression

$$
\left[\begin{array}{c}
\dot{U}_{c} \\
\dot{I}
\end{array}\right]=\left[\begin{array}{cc}
0 & 1 / C \\
-1 / L & -R / L
\end{array}\right]\left[\begin{array}{c}
U_{c} \\
I
\end{array}\right]+\left[\begin{array}{c}
0 \\
U_{r} / L
\end{array}\right]
$$

According to the trajectory section eigenvalue method, the system is linearized and expanded on each time section.

$$
\begin{gathered}
\left\{\begin{array}{c}
\Delta \dot{U}_{c, k}=I_{k} / C_{k}+\Delta I_{k} / C_{k} \\
\Delta \dot{I}_{k}=\Delta U_{c, k} / L_{k}-R_{k} \Delta I_{k} / L_{k} \\
\quad+\left(U_{c, k}-R_{k} I_{k}+U_{r, k}\right) / L_{k}
\end{array}\right. \\
\boldsymbol{A}_{k}=\left[\begin{array}{cc}
0 & 1 / C_{k} \\
-1 / L_{k} & -R_{k} / L_{k}
\end{array}\right]
\end{gathered}
$$

$$
\boldsymbol{B}_{\boldsymbol{k}}=\left[\begin{array}{c}
I_{k} / C_{k} \\
\left(U_{c, k}-R_{k} I_{k}+U_{r, k}\right) / L_{k}
\end{array}\right]
$$

The state increment expression from ${ }^{t_{k}}$ to ${ }^{t_{k+1}}$ :

$$
\Delta \dot{\boldsymbol{X}}_{k}(\Delta t)=\boldsymbol{A}_{k} \Delta \boldsymbol{X}_{k}(\Delta t)+\boldsymbol{B}_{k}
$$

According to Kirchhoff's quantification, $U_{c, k}-R_{k} I_{k}+U_{r, k}=0$ when the circuit is operating at a equilibrium point, $I=0, \boldsymbol{B}_{k}=0$.

$$
\Delta \dot{X}_{k}(\Delta t)=A_{k} \Delta X_{k}(\Delta t)
$$

It can be seen from equation (25) that the dynamic behavior of the system in the minimum range of the balance point is completely determined by the trajectory section eigenvalue. It proves that the equilibrium point eigenvalue is the special point on the trajectory section eigenvalue.

The eigenvalue of system is

$$
\lambda_{k, 12}=-\frac{R_{k}}{2 L_{k}} \pm \sqrt{\left(\frac{R_{k}}{2 L_{k}}\right)^{2}-\frac{1}{L_{k} C_{k}}}
$$

The attenuation factor, resonance frequency and natural oscillation angular frequency of the system at the $\boldsymbol{k}$ time section are respectively:

$$
\begin{gathered}
\alpha_{k}=\frac{R_{k}}{2 L_{k}} \\
\omega_{0, k}=\sqrt{\frac{1}{L_{k} C_{k}}} \\
\omega_{d, k}=\sqrt{\left|\omega_{0, k}^{2}-\alpha_{k}^{2}\right|}
\end{gathered}
$$

\section{TIME-VARYING FACTOR SIMULATION AND StABILITY ONLINE ASSESSMENT FRAMEWORK}

State matrix of second-order resonant circuit consists of $R, L, C$. Give $R, L, C$ different time-varying features to simulate the time-varying factors in the system.

Without considering nonsingularity, an n-order square matrix has $\mathrm{n}$ eigenvalues. A pair of conjugate complex eigenvalues describes an oscillating component with sinusoidal features, a real eigenvalue represents an exponentially decayed or divergent aperiodic component. The real part of the eigenvalue is the instantaneous damping feature $\sigma_{k, i}$, the modulus of the imaginary part of the eigenvalue divided by $2 \pi$ is the instantaneous frequency feature $f_{k, i} 20$. According to Lyapunov's first method, when the real part has a positive value, the system issues an instability warning.

In order to explore the difference between the trajectory section eigenvalue method and the signal processing method in the online stability assessment, the simulation will use wavelet transform, Prony two typical signal analysis methods202119 to identify the response curve of the second-order resonant circuit after being disturbed, track system damping and frequency changes through a sliding time window, alarm when the system 
damping exceeds the threshold. The relevant literature of the three signal analysis methods is very complete, and no further explanation is given here.

\section{SIMULATION}

In the steady state operation of the second-order resonant circuit, the power supply voltage is $10 \mathrm{~V}$, the capacitor voltage is $10 \mathrm{~V}$, and the current in the circuit is $0 \mathrm{~A}$.

\subsection{0n-line Evaluation of Stability of Time- varying Systemss}

Simulate time-varying features. $R=1.5-0.1 \times t$, $L=1+0.3 \times \sin (0.8 \times \mathrm{t}), C=0.05 F$, The voltage source suddenly drops to $6 \mathrm{~V}$ at $0 \mathrm{~s}$ and returns to $10 \mathrm{~V}$ after $0.5 \mathrm{~s}$.

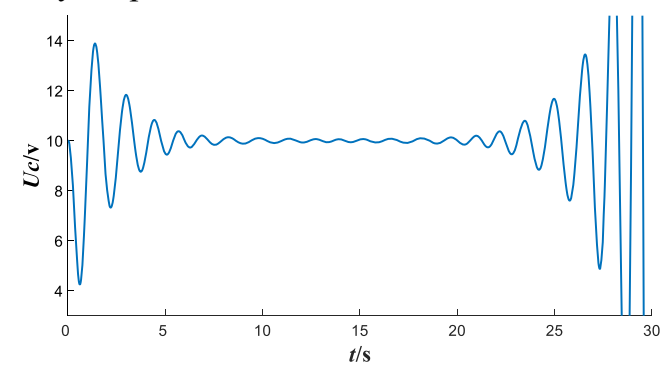

Figure 2 Response waveform of Uc

It can be seen from Fig. 2. that the system has experienced oscillation attenuation, constant amplitude oscillation, divergent oscillation after disturbance, and finally tends to be unstable.

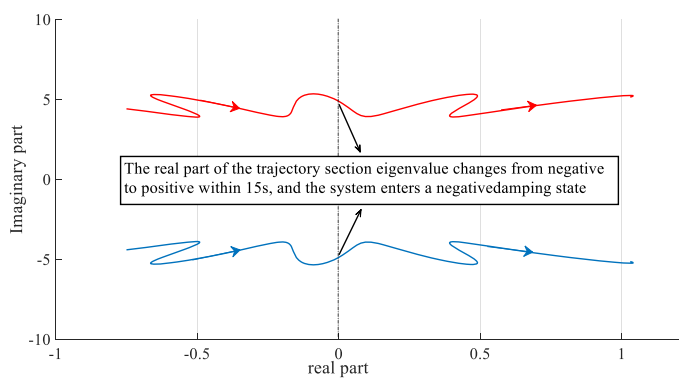

Figure 3 Atlas of TSE

The damping and frequency features are extracted from the eigenvalue sequence, extract the oscillation features by using the Morlet complex wavelets with a wavenumber of 6 and a wavenumber of 12 (time window widening) combined with the wavelet ridge method [19], the frequency resolution is $0.01 \mathrm{~Hz}$. Compare the extraction results of the two methods.

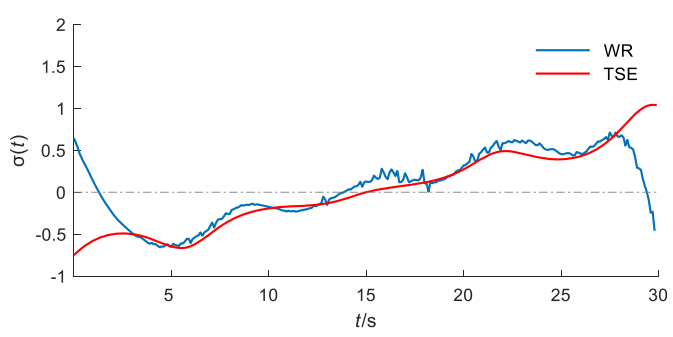

(a) Damping feature when wave number is 6

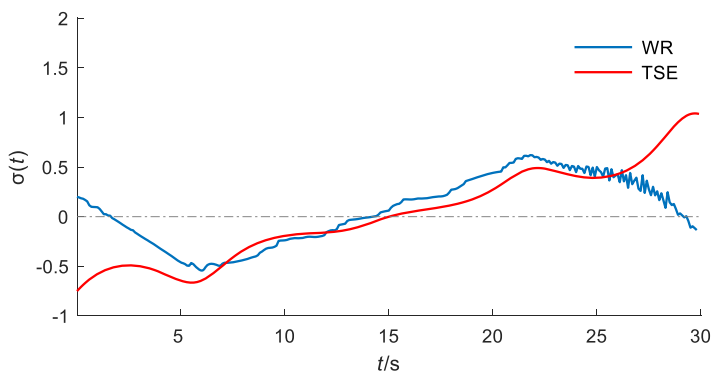

(b) Damping feature when wave number is 12

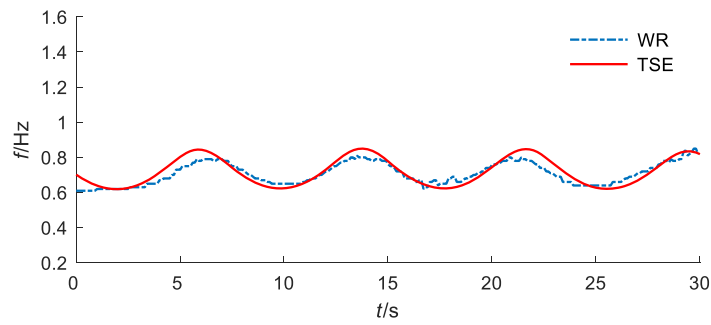

(c) Frequency feature when wave number is 6

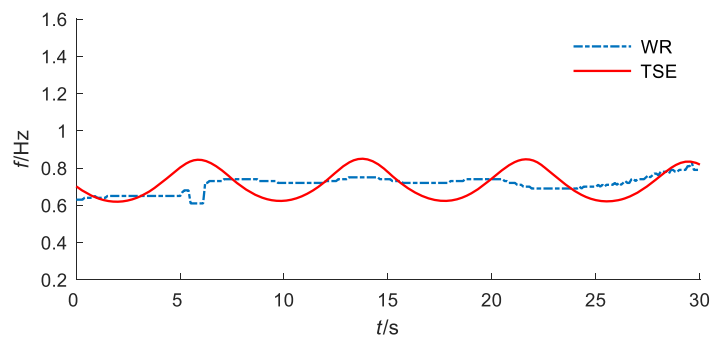

(d) Frequency feature when wave number is 12

Figure 4 Oscillation feature extraction by wavelet ridge method

According to Fig. 4. the wavelet method has obvious limitations. The accuracy of the analysis of system oscillation features is directly related to the length of the time window. For processes with strong time-varying features, the wavelet ridge method has to use an appropriately narrow window to correctly reflect the time-varying oscillation feature. In addition, the existence of the time window leads to the end effect, so the beginning and the end of the curve in Fig. 4. cannot be included in the observation interval.

When evaluating system stability online, the trajectory section eigenvalue method is more sensitive than the signal processing method. The signal processing method completes the oscillation feature acquisition by moving the time window, so the real instability point must be ahead of the operating point. The trajectory section eigenvalue method updates the state matrix by monitoring system parameters, and calculates the eigenvalues in each analysis step to complete the stability evaluation, which is fast and time-short. 


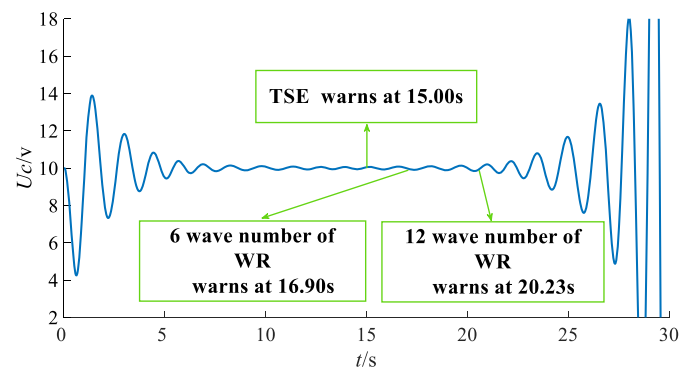

Figure 5 Instability warning of wavelet

According to Fig. 5, the trajectory section eigenvalue method detects that the system damping becomes nonnegative at $15 \mathrm{~s}$, and an instability warning is issued; the Molert complex wavelet with a wave number of 6 issues an instability warning at $16.90 \mathrm{~s}$, and the Molert complex wavelet with a wave number of 12 issues an instability warning at $20.23 \mathrm{~s}$. The trajectory section eigenvalue method can issue an instability warning before the wavelet method.

Fig. 6. compares the extraction results of the system oscillation characteristics by the Prony method and the trajectory section eigenvalue method. The Prony method uses $4 \mathrm{~s}$ and $8 \mathrm{~s}$ sliding time windows to extract the oscillation features, the length of sliding step is $0.01 \mathrm{~s}$.

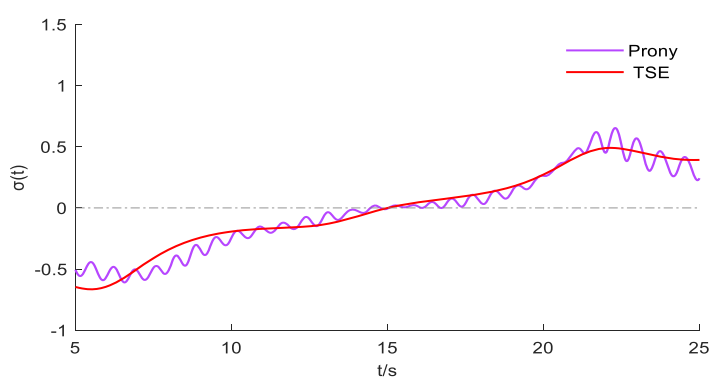

(a) Damping feature extracted by Prony when the window is $4 \mathrm{~s}$

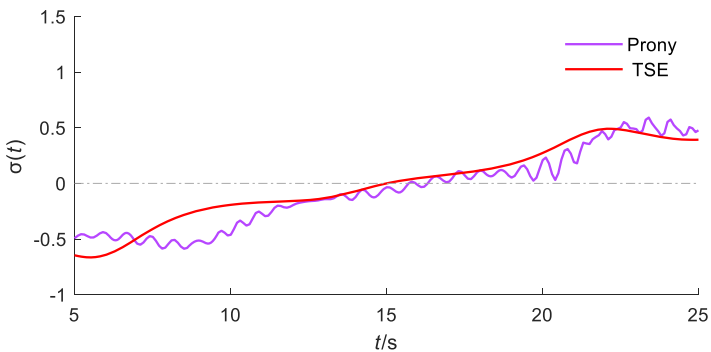

(b) Damping feature extracted by Prony when the window is $8 \mathrm{~s}$

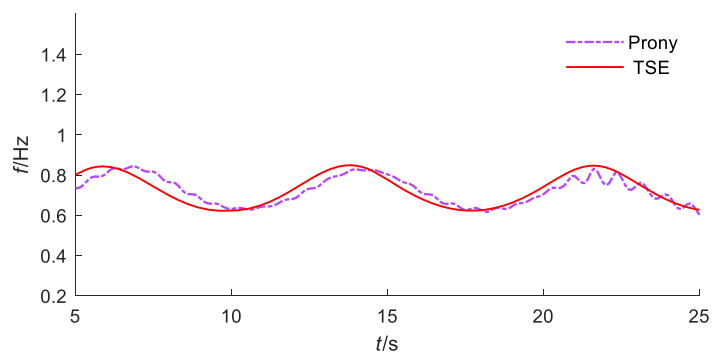

(c) Frequency feature extracted by Prony when the window is $4 \mathrm{~s}$

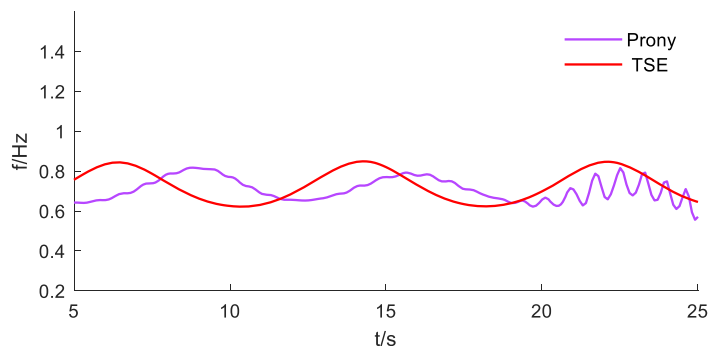

(d) Frequency feature extracted by Prony when the window is $8 \mathrm{~s}$

Figure 6 Oscillation feature extraction of Prony

Intercept the curve from $5 \mathrm{~s}$ to $25 \mathrm{~s}$ at the center of the window to avoid the end effect. In the comparison between the Prony method and the trajectory section eigenvalue method, the Prony method with the $4 \mathrm{~s}$ window can better reflect the damping and frequency feature of the system, while the $8 \mathrm{~s}$ window Prony method can better reflect the damping and frequency feature of the system. The window will produce a large time lag and cannot reflect the time-varying characteristics of the system in time.

Fig. 7. is the instability warning diagram of the Prony method. The system issues an instability warning at $16.48 \mathrm{~s}$, when the time window is $4 \mathrm{~s}$, and the system issues an instability warning at $19.71 \mathrm{~s}$, when the time window is $8 \mathrm{~s}$. They both alarm after $15 \mathrm{~s}$.

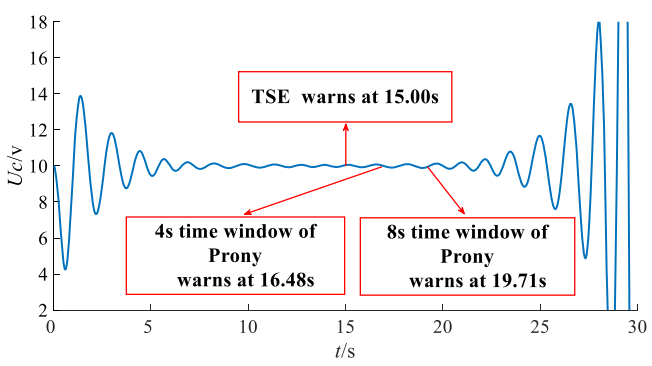

Figure 7 Instability warning of Prony

Table 1 summarizes the alarm time of the two signal processing methods.

TABLE 1 ALARM TIME LIST

\begin{tabular}{ccc}
\hline Method & Window & Alarm Time \\
\hline TSE & none & $15 \mathrm{~s}$ \\
\hline \multirow{2}{*}{ Wavelet } & 6 wave & $16.90 \mathrm{~s}$ \\
\cline { 2 - 3 } & 12 wave & $20.23 \mathrm{~s}$ \\
\hline \multirow{2}{*}{ Prony } & $4 \mathrm{~s}$ & $16.48 \mathrm{~s}$ \\
\cline { 2 - 3 } & $8 \mathrm{~s}$ & $19.71 \mathrm{~s}$ \\
\hline
\end{tabular}

The above simulation shows that the TSE method can quickly track the time-varying oscillation feature of the system by continuously updating the system parameters, constructing the state matrix and calculating the eigenvalues, then judge the stability accurately.

The signal processing method scans the response curve through a sliding time window. Because of the time window, the system instability alarm point will 
always lag behind the actual operating point. The lag time is related to the selection of the signal processing method and the length of the time window.

\subsection{Comparison of Equilibrium Point Eigenvalue and Trajectory Section Eigenvalue Method}

In order to compare the difference between the equilibrium point eigenvalue and the trajectory section eigenvalue more intuitively, we carried out the following simulation.

Simulate time-varying factors, $R=1.5-0.1 \times t$, $L=1, C=0.2$ the voltage source rises to $12 \mathrm{~V}$ in the $5 \mathrm{~s}$, Fig. 10 shows the disturbance response curve.

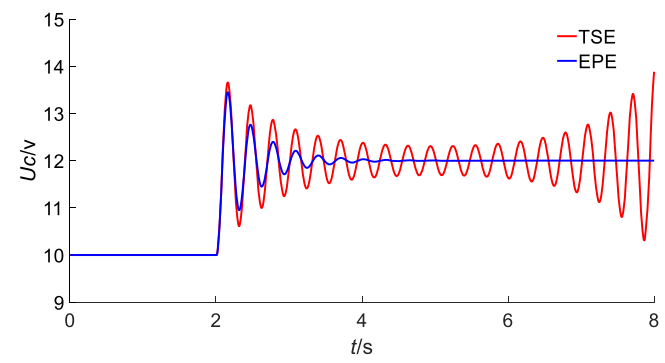

Figure 8 Disturbance response curve

Known from waveform of TSE and Equilibrium Point Eigenvalue (EPE), their assessment of stability is completely different, in order to study the mechanism of this phenomenon, the section extracts the eigenvalue information of TSE and EPE separately at the critical time section, and conducts an in-depth analysis of the dynamic response process to verify the consistency of the two methods with the dynamic behavior of the system.

TABLE 2 EigenVALUeS OF Key SeCtion

\begin{tabular}{|c|c|c|c|c|c|}
\hline \multirow{2}{*}{$\mathrm{t}(\mathrm{s})$} & \multicolumn{2}{|c|}{ EPE } & \multirow{2}{*}{$\mathrm{t}(\mathrm{s})$} & \multicolumn{2}{|c|}{ TSE } \\
\hline & real & imagine & & real & imagine \\
\hline 9 & -1.000 & \pm 8.1035 & 9 & -0.4000 & \pm 8.1551 \\
\hline 12 & -1.000 & \pm 8.1035 & 12 & -0.2000 & \pm 8.1625 \\
\hline 15 & -1.000 & \pm 8.1035 & 15 & 0.0000 & \pm 8.1650 \\
\hline 18 & -1.000 & \pm 8.1035 & 18 & 0.2000 & \pm 8.1625 \\
\hline
\end{tabular}

According to Table 2, the eigenvalue following the method of EPE is $-1.000 \pm 8.1035 \mathrm{i}$ through the whole process, and the real part of the eigenvalue is always -1 . The whole process has positive damping feature, and the system should oscillate attenuated and return to the steady state of equilibrium.

However, the trajectory of the numerical simulation shows that the oscillation is excited again after attenuation and begins to diverge. Obviously, it does not match the conclusion of the EPE method, the EPE method is invalid in this situation. According to the TSE method, the system updates the state matrix one time section by time, the real part of the eigenvalue undergoes a process from negative to positive, and the damping feature of the system changes from negative to positive, which explains the phenomenon that the simulation trajectory first attenuates the oscillation and then diverges.

\section{CONCLUSION}

The TSE method combines the model and the trajectory, uses piecewise linearization to extend the EPE to any time section of the whole process of the system response, it solves the time-varying and nonlinear problems that plague the EPE method, shows the influence mechanism of time-varying factors on system stability. Based on clarifying the theory of TSE, this paper establishes a priori model and simulates time-varying factors, and applies TSE method and signal processing method to analyze the perturbed oscillation curve of the system model, proving that the TSE method can reflect the timevarying feature better than the signal processing method, can issue an instability warning before the signal processing method. Through the comparison between TSE method and traditional signal processing method, this paper demonstrates the time and speed advantages of TSE in online stability evaluation, which is of great significance to engineering applications. The TSE method deserves to be further studied and promoted.

\section{REFERENCES}

1. Z. Bin and Y. Xue, "A method to extract instantaneous features of low frequency oscillation based on trajectory section eigenvalues," in Journal of Modern Power Systems and Clean Energy, vol. 7, no. 4, pp. 753-766, July 2019, doi: $10.1007 /$ s40565019-0556-z.

2. J. Thapar, V. Vittal, W. Kliemann and A. A. Fouad, "Application of the normal form of vector fields to predict interarea separation in power systems," in IEEE Transactions on Power Systems, vol. 12, no. 2, pp. 844-850, May 1997, doi: 10.1109/59.589716.

3. J. J. Sanchez-Gasca et al., "Inclusion of higher order terms for small-signal (modal) analysis: committee report-task force on assessing the need to include higher order terms for small-signal (modal) analysis," in IEEE Transactions on Power Systems, vol. 20, no. 4, pp. 1886-1904, Nov. 2005, doi: 10.1109/TPWRS.2005.858029.

4. LI Xun, GONG Qingwu, QIAO Hui, et al. Nonparametric high-order spectral analysis method for nonlinear correlation of low-frequency oscillation modes[J]. Proceedings of the CSEE,2013,33(7):6168,18 .

5. ZHANG Jing, WEN Jin - yu, CHENG Shi - jie. Normal forms of vector fields based analysis of power system angle and voltage stability characteristic $[\mathrm{J}]$. Automation of Electric Power Systems, 2006,30(12):0.

6. N. Pariz, H. M. Shanechi and E. Vaahedi, "Explaining and validating stressed power systems behavior using modal series," 2003 IEEE Power Engineering Society General Meeting (IEEE Cat. No.03CH37491), Toronto, ON, Canada, 2003, pp. 1285-1285, doi: 10.1109/PES.2003.1267333.

7. LIU Hongchao, LI Xingyuan, HAO Wei, et al. Nonlinear modal analy sis for HVDC/AC power sy 
stems. Automation of Electric Power Systems, 2006, 30(18):8-12.

8. XUE Yusheng, PAN Xueping, ZHANG Guorui, et al.Oscillation mode analysis including full nonlinearity of time-varying systems. Automation of Electric Power Systems,2008, 32(18):1-7.

9. PAN Xueping, XUE Yusheng, et al. Analytical Calculation of Power System Trajectory Eigenvalues and Its Error Analysis[J]. Automation of Electric Power Systems, 2008, 32(19):10-14.

10. NI Jingmin, SHEN Chen, TAN Wei, et al. A Coherence Identifying Method Based on Linearization at Non-equilibrium Point[J]. Automation of Electric Power Systems, 2010, 34(20):7-12.

11. TAN Wei, SHEN Chen, LI Ying, et al. A Generator Groups Identification Method Based on Trajectory Eigenvalues $[\mathrm{J}]$. Automation of Electric Power Systems, 2010, 34(1):8-14.

12. TAN Wei, SHEN Chen, NI Jingmin, et al. Analysis and Application of Power Control Sensitivity Based on Trajectory Eigenvalues[J]. Automation of Electric Power Systems, 2010, 34(18):1-5.

13. TAN Wei, SHEN Chen, LIU Feng, et al. A Practical Criterion for Trajectory Eigenvalues Based Transient Stability Analysis[J]. Automation of Electric Power Systems, 2012, 36(16):14-19.

14. XUE Yusheng, HAO Lili, et al. Annotation for FEP and DSP in Terms of Trajectory Section
Eigenvalues[J]. Automation of Electric Power Systems, 2010, 34(12):1-7.

15. YUAN You, KANG Jitao, WANG Delin, et al. Judgment of System Transient Stability and Disturbance Type Screening Method Based on Trajectory Eigenvalue. Power System Technology[J], 2018,42(10):3330-3338.

16. LU Dandan, YANG Feiyan, et al. A Contingency Screening Method Based on Trajectory Eigenvalues[J]. Automation of Electric Power Systems, 2013, 37(5):38-43.

17. XUE Yusheng, HAO Sipeng, et al. A Review of Analysis Methods for Low-frequency Oscillations[J]. Automation of Electric Power Systems, 2009, 33(3):1-8.

18. Lala P. An Introduction to Logic Circuit Testing[M]. Morgan \& Claypool, 2008.

19. NI Yi-xin, CHEN Shou-sun. ZHANG Bao-lin. Dynamic power system theory and analysis[M]. Beijing: Tsinghua University Press, 2002.

20. HAO Sipeng, XUE Yusheng, et al. Trajectory Eigenvalues Analysis Time Variant Oscillation Characters[J]. Automation of Electric Power Systems, 2009, 33(6):1-5.

21. HU Haoming, ZHENG Wei, XU Wei, et al. Comparison of the applicability of Prony and HHT algorithms for on-line identification of lowfrequency oscillation[J]. Power System Protection and Control, 2013, 41(14):33-40. 